\title{
Current detection of superradiance and induced entanglement of double quantum dot excitons
}

\author{
Y. N. Chen ${ }^{1}$, D. S. Chuu ${ }^{1}$, and T. Brandes ${ }^{2}$ \\ ${ }^{1}$ Department of Electrophysics, National Chiao-Tung University, Hsinchu 30050, Taiwan \\ ${ }^{2}$ Department of Physics, UMIST, P.O. Box 88, Manchester, M60 1QD, U.K.
}

(Dated: May 16, 2018)

\begin{abstract}
We propose to measure the superradiance effect by observing the current through a semiconductor double-dot ststem. An electron and a hole are injected separately into one of the quantum dots to form an exciton and then recombine radiatively. We find that the stationary current shows oscillatory behavior as one varies the inter-dot distance. The amplitude of oscillation can be increased by incorporating the system into a microcavity. Furthermore, the current is suppressed if the dot distance is small compared to the wavelength of the emitted photon. This photon trapping phenomenon generates the entangled state and may be used to control the emission of single photons at predetermined times.
\end{abstract}

PACS: 03.65.Ud, 03.67.Mn, 42.50.Fx, 71.35.-y

Since Dicke proposed the phenomenon of superradiance [1, the coherent radiation phenomena for atomic systems were intensively investigated. In semiconductor systems, the electron-hole pair is naturally a candidate for examining the spontaneous emission. The decay rate of the exciton is superradiantly enhanced by a factor of $(\lambda / d)^{2}$ for a $2 \mathrm{D}$ exciton-polariton system [2], where $\lambda$ is the wave length of the emitted photon and $d$ is the lattice constant of the thin film. In the past decades, the superradiance of excitons in these quantum well structures has been investigated intensively [3]. For lower dimensional systems, the decay rate of the exciton is enhanced by a factor of $\lambda / d$ in a quantum wire [4]. In the quantum dots, although the decay rate is shown to be proportional to $\mathrm{R}^{2.1}[\underline{5}]$ which confirms the theoretical prediction [6], acceptable experimental data on quantum dot superradiance are still not plentiful owing to the difficulty of techniques in observing the enhanced spontaneous emission optically.

In recent years, great attention has been focused on the entanglement issue since generation of highly entangled states is one of the fundamental requirements for quantum information processing [7]. Lots of works have been associated with quantum-optic and atomic systems 8 . However, due to the scalability of the quantum processor, solid-state realizations of such phenomena are the favored choices [9]. In fact, the superradiance effect can also generate entanglement by spontaneous emission. Therefore, a different way is proposed to observe the superradiant effect and generate the entangled states in this work. By injecting electrons and holes into one of the quantum dots of a double dot system and controlling the gate voltage of one of the dots, a photon is then generated by the coherent recombination of the exciton. This process not only allows one to determine the superradiant effect by measuring the stationary current, but also induces the entangled states in this double-dot system.

In our model, we consider a p-i-n junction, similar to the device proposed by Benson et al [10], but with two spatially separated quantum dots. The novel feature here is the dissipative creation of entanglement over relatively large distances, and its readout via the stationary current The device structure is shown in Fig.1.

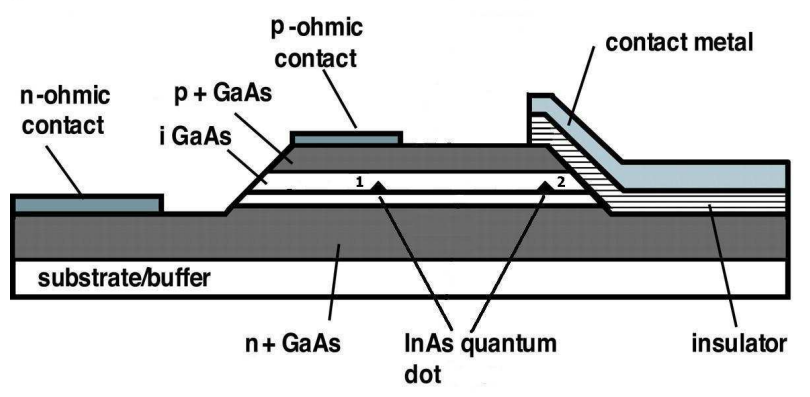

FIG. 1: Proposed device structure. Two InAs quantum dots are embedded in a $p-i$ - $n$ junction. Above dot 2 is a metal gate, which control the energy gap and orientation of the dipole.

One of the obstacles in measuring superradiance between the quantum dots comes from the random size of the dots which result in a random distribution of energy gap and thus diminishes the coherent radiation. This can be overcome by constructing a gate voltage over one of the quantum dots. The energy gap and the orientation of the dipole moments of one of the quantum dots, thus can be controlled well. Both the hole and electron reservoirs are assumed to be in thermal equilibrium. For the physical phenomena we are interested in, the current is conducted through dot 1 only, and the fermi level of the $p(n)$-side hole (electron) is slightly lower (higher) than the hole (electron) subband in the dot. After a hole is injected into the hole subband in the quantum dot, the $n$-side electron can tunnel into the exciton level because of the Coulomb interaction between the electron and hole. In our calculation, we also neglect the Forster process which may have some influences on the results if the two dots are close to each other. The validity of this assumption will be discussed later. Thus, we may assume four dot states $|0\rangle=|0, h ; 0,0\rangle,\left|U_{1}\right\rangle=|e, h ; 0,0\rangle$, $\left|U_{2}\right\rangle=|0,0 ; e, h\rangle$, and $|D\rangle=|0,0 ; 0,0\rangle$, where $|0, h ; 0,0\rangle$ 
means there is one hole in dot 1 and $|0,0 ; 0,0\rangle$ represents the ground state with no hole and electron in the quantum dots. The exciton states $|e, h ; 0,0\rangle$ (in dot 1) can be converted to $|0,0 ; e, h\rangle$ (in dot 2 ) through the excitonphoton interactions. One might argue that one can not neglect the state $|e, 0 ; 0,0\rangle$ for real device since the tunable variable is the applied voltage. However, this can be resolved by fabricating a thicker barrier on the electron side so that there is little chance for an electron to tunnel in advance. By transforming $\left|U_{1}\right\rangle$ and $\left|U_{2}\right\rangle$ into Dicke states: $\left|S_{0}\right\rangle=\frac{1}{\sqrt{2}}\left(\left|U_{1}\right\rangle-\left|U_{2}\right\rangle\right)$ and $\left|T_{0}\right\rangle=$ $\frac{1}{\sqrt{2}}\left(\left|U_{1}\right\rangle+\left|U_{2}\right\rangle\right)$, we can now define the dot-operators $\hat{n_{S}} \equiv\left|S_{0}\right\rangle\left\langle S_{0}\left|, \hat{n_{T}} \equiv\right| T_{0}\right\rangle\left\langle T_{0}\left|, \hat{n_{D}} \equiv\right| D\right\rangle\langle D|, \hat{p_{s}} \equiv$ $\left|S_{0}\right\rangle\left\langle D\left|, \hat{p_{T}} \equiv\right| T_{0}\right\rangle\langle D|, \hat{s}_{U_{1}}^{\wedge} \equiv \frac{1}{\sqrt{2}}\left(|0\rangle\left\langle S_{0}|+| 0\right\rangle\left\langle T_{0}\right|\right)$, $\hat{s_{D}} \equiv|0\rangle\langle D|$. The total hamiltonian $H$ of the system consists of three parts: $H_{0}$ (dot, photon bath $H_{p}$, electron (hole) reservoirs $H_{\text {res }}$ ), $H_{T}$ (dot-photon coupling), and the dot-reservoir coupling $H_{V}$ :

$$
\begin{aligned}
H= & H_{0}+H_{T}+H_{V} \\
H_{0}= & \varepsilon_{U} \hat{n_{S}}+\varepsilon_{U} \hat{n_{T}}+\varepsilon_{D} \hat{n_{D}}+H_{p}+H_{\text {res }} \\
H_{T}= & \sum_{\mathbf{k}} \frac{1}{\sqrt{2}} g\left\{D _ { \mathbf { k } } b _ { \mathbf { k } } \left[\hat{p_{S}}\left(1+e^{i \mathbf{k} \cdot \mathbf{r}}\right)\right.\right. \\
& \left.\left.+\hat{p}_{T}\left(1-e^{i \mathbf{k} \cdot \mathbf{r}}\right)\right]+c . c .\right\} \\
= & g\left(\hat{p}_{S} X_{S}+\hat{p}_{S}^{\dagger} X_{S}^{\dagger}+\hat{p}_{T} \overline{X_{T}}+\hat{p}_{T}^{\dagger} \overline{X_{T}}{ }^{\dagger}\right) \\
H_{p}= & \sum_{\mathbf{k}} \omega_{\mathbf{k}} b_{\mathbf{k}}^{\dagger} b_{\mathbf{k}} \\
H_{V}= & \sum_{\mathbf{q}}\left(V_{\mathbf{q}} c_{\mathbf{q}}^{\dagger} \hat{s}_{U_{1}}+W_{\mathbf{q}} d_{\mathbf{q}}^{\dagger} \hat{s}_{D}+c . c .\right) \\
H_{r e s}= & \sum_{\mathbf{q}} \varepsilon_{\mathbf{q}}^{U} c_{\mathbf{q}}^{\dagger} c_{\mathbf{q}}+\sum_{\mathbf{q}} \varepsilon_{\mathbf{q}}^{D} d_{\mathbf{q}}^{\dagger} d_{\mathbf{q}} .
\end{aligned}
$$

In the above equation, $b_{\mathbf{k}}$ is the photon operator, $g D_{\mathbf{k}}$ is the dipole coupling strength, $\mathbf{r}$ is the position vector between two quantum dot, $X_{S}=\sum_{\mathbf{k}}\left(1+e^{i \mathbf{k} \cdot \mathbf{r}}\right) D_{\mathbf{k}} b_{\mathbf{k}}$, $\overline{X_{T}}=\sum_{\mathbf{k}}\left(1-e^{i \mathbf{k} \cdot \mathbf{r}}\right) D_{\mathbf{k}} b_{\mathbf{k}}$, and $c_{\mathbf{q}}$ and $d_{\mathbf{q}}$ denote the electron operators in the left and right reservoirs, respectively. The dipole approximation is not used in our calculation since we keep the full $e^{i \mathbf{k} \cdot \mathbf{r}}$ terms in the operators $X_{S}$ and $\overline{X_{T}}$. Here, $g$ is a constant with a unit of the tunneling rate. The couplings to the electron and hole reservoirs are given by the standard tunnel hamiltonian $H_{V}$, where $V_{\mathbf{q}}$ and $W_{\mathbf{q}}$ couple the channels $\mathbf{q}$ of the electron and the hole reservoirs. If the couplings to the electron and the hole reservoirs are weak, then it is reasonable to assume that the standard Born-Markov approximation with respect to these couplings is valid. In this case, one can derive a master equation from the exact time-evolution of the system. The equations of motion can be expressed as (cp. [23])

$$
\begin{aligned}
& \left\langle\hat{n_{\sigma}}\right\rangle_{t}-\left\langle\hat{n_{\sigma}}\right\rangle_{0}=-i g \int_{0}^{t} d t^{\prime}\left\{\left\langle\hat{\left.p_{\sigma}\right\rangle_{t^{\prime}}}-\left\langle\hat{\left.p_{\sigma}^{\dagger}\right\rangle_{t^{\prime}}}\right\}\right.\right. \\
& +\Gamma_{U} \int_{0}^{t} d t^{\prime}\left(1-\left\langle\hat{n_{S}}\right\rangle_{t^{\prime}}-\left\langle\hat{n_{T}}\right\rangle_{t^{\prime}}-\left\langle\hat{n_{D}}\right\rangle_{t^{\prime}}\right) \\
& \left\langle\hat{n_{D}}\right\rangle_{t}-\left\langle\hat{n_{D}}\right\rangle_{0}=-i g \int_{0}^{t} d t^{\prime}\left\{\left\langle\hat{p_{S}}\right\rangle_{t^{\prime}}-\left\langle\hat{p_{S}^{\dagger}}\right\rangle_{t^{\prime}}+\left\langle\hat{p_{T}}\right\rangle_{t^{\prime}}\right. \\
& \left.-\left\langle\hat{p_{T}^{\dagger}}\right\rangle_{t^{\prime}}\right\}-2 \Gamma_{D} \int_{0}^{t} d t^{\prime}\left\langle\hat{n_{D}}\right\rangle_{t^{\prime}} \\
& \left\langle\hat{p_{S}}\right\rangle_{t}-\left\langle\hat{\left.p_{S}\right\rangle_{t}^{0}}=-\Gamma_{D} \int_{0}^{t} d t^{\prime} e^{i \varepsilon\left(t-t^{\prime}\right)}\left\langle X_{t} X_{t^{\prime}}^{\dagger} \widetilde{P_{S}}\left(t^{\prime}\right)\right\rangle_{t^{\prime}}\right. \\
& -i g \int_{0}^{t} d t^{\prime} e^{i \varepsilon\left(t-t^{\prime}\right)}\left\{\left\langle\hat{n}_{S} X_{t} X_{t^{\prime}}^{\dagger}\right\rangle_{t^{\prime}}\right. \\
& \left.-\left\langle\hat{n_{D}} X_{t^{\prime}}^{\dagger} X_{t}\right\rangle_{t^{\prime}}\right\}
\end{aligned}
$$

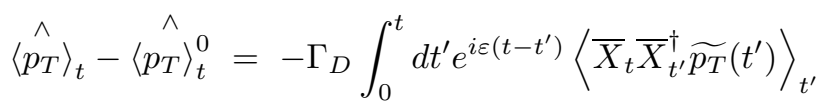

$$
\begin{aligned}
& -i g \int_{0}^{t} d t^{\prime} e^{i \varepsilon\left(t-t^{\prime}\right)}\left\{\left\langle n_{T}^{\wedge} \bar{X}_{t} \bar{X}_{t^{\prime}}^{\dagger}\right\rangle_{t^{\prime}}\right. \\
& \left.-\left\langle\hat{n}_{D} \bar{X}_{t^{\prime}}^{\dagger} \bar{X}_{t}\right\rangle_{t^{\prime}}\right\}
\end{aligned}
$$

, where the index $\sigma=S$ or $T, \Gamma_{U}=\pi \sum_{\mathbf{q}} V_{\mathbf{q}}^{2} \delta\left(\varepsilon_{U}-\varepsilon_{\mathbf{q}}^{U}\right)$ , $\Gamma_{D}=\pi \sum_{\mathbf{q}} W_{\mathbf{q}}^{2} \delta\left(\varepsilon_{D}-\varepsilon_{\mathbf{q}}^{D}\right)$, and $\varepsilon=\varepsilon_{U}-\varepsilon_{D}$ is the energy gap of the quantum dot exciton. Here, $\widetilde{p_{S}}\left(t^{\prime}\right)=$ $p_{S} e^{i \varepsilon t} X_{t^{\prime}}, \widetilde{p_{T}}\left(t^{\prime}\right)=p_{T} e^{i \varepsilon t} \bar{X}_{t^{\prime}}$, and $X_{t^{\prime}}\left(\bar{X}_{t^{\prime}}\right)$ denotes the time evolution of $X(\bar{X})$ with $H_{p}$. The expectation value $\left\langle p_{\sigma}^{\dagger}\right\rangle_{t}^{0}$ describes the decay of an initial polarization of the system and plays no role for the stationary current. Therefore, we shall assume the initial expectation value of $\hat{p}_{\sigma}^{(\dagger)}$ vanishes at time $t=0$.

As can be seen from Eq. (2), there are terms like $\left\langle\hat{n}_{\sigma} X_{t} X_{t^{\prime}}^{\dagger}\right\rangle_{t^{\prime}}$ which contain products of dot operators and photon operators. If we are interested in small coupling parameters here, a decoupling of the reduced density matrix $\widetilde{\rho_{\sigma}}\left(t^{\prime}\right)$ can be used, $\widetilde{\rho_{\sigma}}\left(t^{\prime}\right) \approx \rho_{p h}^{0} T r_{p h} \widetilde{\rho_{\sigma}}\left(t^{\prime}\right)$. Products of these operators can then be obtained. For spontaneous emission, the photon bath is assumed to be in equilibrium. The expectation values $\left\langle X_{t} X_{t^{\prime}}^{\dagger}\right\rangle_{0} \equiv$ $C\left(t-t^{\prime}\right)$ and $\left\langle\bar{X}_{t} \bar{X}_{t^{\prime}}^{\dagger}\right\rangle_{0} \equiv \bar{C}\left(t-t^{\prime}\right)$ are functions of the time difference only. We can now define the Laplace transformation for real $z$,

$$
\begin{aligned}
& C_{\varepsilon}(z) \equiv \int_{0}^{\infty} d t e^{-z t} e^{i \varepsilon t} C(t) \\
& n_{\sigma}(z) \equiv \int_{0}^{\infty} d t e^{-z t}\left\langle\hat{n}_{\sigma}\right\rangle_{t} \text { etc., } z>0
\end{aligned}
$$

and transform the whole equations of motion into $z$ space. The tunnel current $\widehat{I}$ can be defined as the 
change of the occupation of $\hat{n_{D}}$ and is given by $\widehat{I} \equiv$ $i g \sum_{\sigma}\left(\hat{p}_{\sigma}-{\hat{p_{\sigma}}}^{\dagger}\right)$, where we have set the electron charge $e=1$ for convenience. The time dependence of the expectation value $\langle\hat{I}\rangle_{t}$ can be obtained by performing the inverse Laplace transformation. For time $t \rightarrow \infty$, the result is

$$
\langle\rangle_{t \rightarrow \infty}=\frac{4 g^{2} \gamma_{+} \gamma_{-}}{\gamma_{-}+\gamma_{+}\left[1+2 \gamma_{-}\left(g^{2} / \Gamma_{D}+g^{2} / \Gamma_{U}+\Gamma_{D}\right)\right]}
$$

, where $g^{2} \gamma_{+}$and $g^{2} \gamma_{-}$are the superradiant and subradiant decay rate of the exciton, respectively. 11] The derivation of the current equation is closely analogous to the spontaneous emission of phonons in double dots [12], in which the correlation functions $\left\langle X_{t} X_{t^{\prime}}^{\dagger}\right\rangle_{0}$ is given by the electron-phonon interaction.

The corresponding decay rate for superradiant and the subradiant channels is given by

$$
g^{2} \gamma_{ \pm}=\gamma_{0}\left(1 \pm \frac{\sin \left(2 \pi d / \lambda_{0}\right)}{2 \pi d / \lambda_{0}}\right)
$$

where $d$ is the inter-dot distance and $\gamma_{0}$ is the exciton decay rate in a quantum dot. To display the dependence of the stationary current through the quantum dot on the dot distance $d$, we present the results of two identical quantum dots in Fig. 2. In plotting

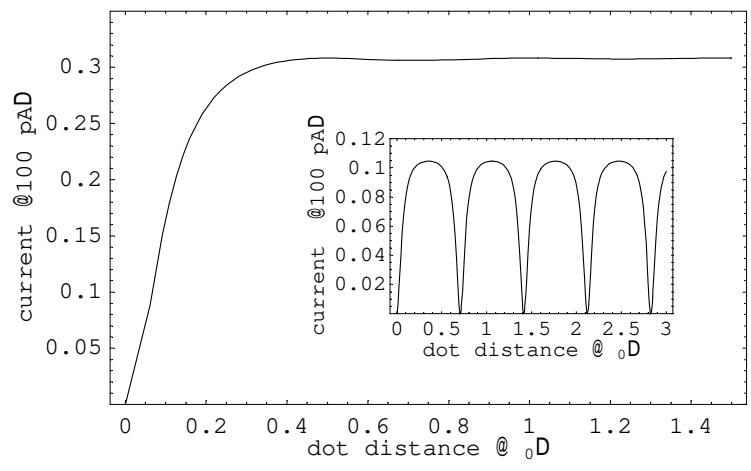

FIG. 2: Stationary tunnel current, Eq. (4), as a function of dot distance $d$. The interference effect is seen clearly (inset) by incorporating the system inside a rectangular microcavity. The vertical and horizontal units are $100 \mathrm{pA}$ and $\lambda_{0}$, respectively.

the figure, the current is in terms of $100 \mathrm{pA}$. Furthermore, the tunneling rates, $\Gamma_{U}$ and $\Gamma_{D}$, are assumed to be equal to $0.2 \gamma_{0}$ and $\gamma_{0}$, respectively. Here, a value of $1 / 1.3 \mathrm{~ns}$ for the free-space quantum dot decay rate $\gamma_{0}$ is used in our calculations 13. As shown in Fig. 2, the current is suppressed as the dot distance $d$ is much smaller than the wavelength $\left(\lambda_{0}\right)$ of the emitted photon. This corresponds to the trapping state in the twoion system. As long as we choose only one of the dots to be coupled to reservoirs, the generated photon is reabsorbed immediately by the other dot and vice versa. The current is then blocked by this exchange process. For small rates limit $\left(g^{2} \gamma_{ \pm}\right)$one can approximate equation (4) by $I \approx 4\left[1 / g^{2} \gamma_{-}+1 / g^{2} \gamma_{+}\right]^{-1}$. The rates $\Gamma_{U, D}$ drop out completely and the current is only determined by the (smaller) radiative decay rates. In this approximate form, the current looks identical to the expression for the conductance $G \propto\left[1 / \Gamma_{L}+1 / \Gamma_{R}\right]^{-1}$ from a left lead through a single level to a right lead with tunnel rates $\Gamma_{L, R}$. This implies that the superradiant and the subradiant channel are in series (and not in parallel) in this limit. This is because once the exciton is formed in $\operatorname{dot} 1$, time evolution of this state is proportional to $e^{-g^{2} \gamma_{+} t}+e^{-g^{2} \gamma_{-} t}$ not $e^{-g^{2}\left(\gamma_{+}+\gamma_{-}\right) t}[14$. It means the two decay channels in our system are not in parallel. For long time behavior $t \rightarrow \infty$ and $\gamma_{+}>>\gamma_{-}$, the function $e^{-g^{2} \gamma_{+} t}+e^{-g^{2} \gamma_{-} t}$ approaches the limit of $e^{-g^{2} \gamma_{-} t}$, which is identical to the same limit of the function $e^{-\frac{g^{2} \gamma_{+} \gamma_{-}}{\gamma+\gamma_{-}} t}$ (in series).

Similar to the two-ion superradiance 11, the current also exhibits oscillatory behavior as a function of dot distance. To observe the interference effect clearly, one may incorporate the system inside a microcavity since semiconductor cavities with strong electron-photon coupling have been realised experimentally by, e.g., Gérard et al. [15]. Reduction of the allowed $k$-state is expected to increase the magnitude of the oscillation. For example, if the system is placed inside a rectangular microcavity with length $\lambda_{0}$, the decay rate for the two channels can be worked out straightforwardly:

$$
g^{2} \gamma_{c a v, \pm}=\frac{\gamma_{0}}{\pi}\left|1 \pm e^{i 2 \pi d /\left(\sqrt{2} \lambda_{0}\right)}\right|^{2} .
$$

The stationary current is plotted in the inset of Fig. 2, where a perfect (lossless) cavity is assumed. As we mentioned above, the amplitude of oscillation is larger than that in free space. However, the oscillation period is not half of the wavelength, but $\lambda_{0} / \sqrt{2}$. This is because the interference term is only influenced by the wave vector in the unconfined direction. Excluding the contributions from fundamental cavity modes, the effective wave vector can be expressed as

$$
k_{e f f}=\sqrt{\left(\frac{2 \pi}{\lambda_{0}}\right)^{2}-2 *\left(\frac{\pi}{\lambda_{0}}\right)^{2}}=\frac{k_{0}}{\sqrt{2}} .
$$

The oscillation period of the decay rate and the current is therefore increased by a factor of $\sqrt{2}$.

In Fig. 3, we plot the expectation value of $n_{S} \quad\left(n_{T}\right)$ as a function of the dot distance. The maximum entangled state $\left(\left|S_{0}\right\rangle\right)$ is reached as $d<<\lambda_{0}$. This is remarkable as the steady state is independent of the initial state. The entanglement is induced by the cooperative decoherence in the system. In a recent paper by Schnider et al. [16], the authors consider the behavior of an ion trap with 
all ions driven simultaneously and coupled collectively to a heat bath. They also found that the steady state of the ion trap can exhibit quantum entanglement. However, the concurrence of their system is below the value of unity (maximum entanglement). On the contrary, in our system the maximum entangled state can be generated by tuning the band gap of dot 2 (linear stark effect), i.e. control the on/off of the superradiance. Another advantage of our scheme is shown in the inset of Fig. 3. If the double-dot system is incorporated inside a rectangular microcavity, the maximum entangled states repeat as a function of inter-dot distance. This means even for remote separation, the entanglement can still be achieved. The reason can be attributed to that the creation of entanglement in our model is governed by the interaction with a common heat bath, while conventional creation of entanglement depends on the direct interaction between two subsystems [17]. When two dots are coupled to the common photon fields, the collective decay process drives the system into the entangled states. The novel feature of the effect predicted here is that entanglement in fact can be controlled electrically (without applying a laser field) and read out in the form of a transport property, i.e., the electron current (as a function of the dot distance or, alternatively, the cavity length).

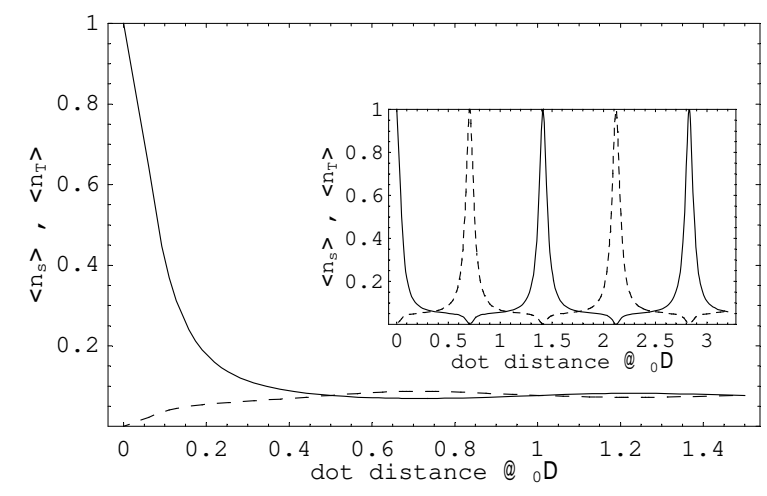

FIG. 3: Occupation probability of the entangled states $n_{S}$ (solid line) and $n_{T}$ (dashed line). The inset shows the results inside a rectangular microcavity.
Another possible application of this effect is that by tuning the coherence of the dots, one can control the emission of single photon at predetermined times, which is important for the field of quantum information technology. However, one should note that the biexciton effect is not included in our present model. Therefore, a low injection limit is required in the experiment [18]. One might argue that for small inter-dot distance the Forster process may play some role in our system $[19]$; nevertheless, this only causes small energy splitting between state $\left|S_{0}\right\rangle$ and $\left|T_{0}\right\rangle$. Comparing to the large energy difference in the III-V semiconductor material, its effect on the decay rate $g^{2} \gamma_{ \pm}$is negligible. As for the problem of dissipation, decoherence due to interaction with other bosonic excitations (phonons and electron-hole pairs in the leads) is inevitable but can in principle be (partly) controlled by variation of the dot energies, or control of the mechanical degree of freedom [20]. In addition, scattering due to impurities are negligible since there is no interdot transport in our system.

In conclusion, we have proposed a method of detecting the superradiant effect in a semiconductor doubledot system. By incorporating the InAs quantum dots between a $p-i-n$ junction, the superradiant effect on the stationary tunnel current can be examined by tuning the band gap of the quantum dot. Moreover, the interference effects between two dots can be seen more clearly by incorporating the system inside a microcavity. The oscillation period of the decay rate and current is also increased because of the microcavity. Besides, the maximum entangled state is induced as the inter-dot distance is much smaller than the wavelength of the emitted photon. Our model provides a new way to generate the entanglement in solid-state systems.

We would like to thank to Prof. Y. C. Lee at SUNYAB for helpful discussions. This work is supported partially by the National Science Council, Taiwan under the grant number NSC 91-2112-M-009-012.
[1] R. H. Dicke, Phys. Rev. 93, 99 (1954).

[2] K. C. Liu and Y. C. Lee, Physica 102A, 131 (1980); Y. N. Chen and D. S. Chuu, Phys. Rev. B 61, 10815 (2000).

[3] J. Knoester, Phys. Rev. Lett. 68, 654 (1992); D. S. Citrin, Phys. Rev. B 47, 3832 (1993); D. Ammerlahn, J. Kuhl, B. Grote, S. W. Koch, G. Khitrova, and H. Gibbs, Phys. Rev. B 62, 7350 (2000).

[4] A. L. Ivanov and H. Haug, Phys. Rev. Lett. 71, 3182 (1993); Y. Manabe, T. Tokihiro, and E. Hanamura, Phys. Rev. B 48, 2773 (1993); Y. N. Chen and D. S. Chuu, T. Brandes, and B. Kramer, Phys. Rev. B 64, 125307 (2001).

[5] A. Nakamura, H. Yamada, and T. Tookizaki, Phys. Rev. B 40, 8585 (1989).
[6] E. Hanamura, Phys. Rev. B 38, 1228 (1988); F. C. Spano, J. R. Kuklinski, and S. Mukamel, Phys. Rev. Lett. 65, 211 (1990).

[7] C. H. Bennett and D. P. DiVincenzo, Nature (London) 404, 247 (2000).

[8] T. Pellizzari, S. A. Gardiner, J. I. Cirac, and P. Zoller, Phys. Rev. Lett. 75, 3788 (1995); J. I. Cirac and P. Zoller, Phys. Rev. Lett. 74, 4091 (1995); K. Molmer and A. Sorensen, Phys. Rev. Lett. 82, 1835 (1999).

[9] A.T. Costa, Jr. and S. Bose, Phys. Rev. Lett. 87, 277901 (2001); W.D. Oliver, F. Yamaguchi, and Y. Yamamoto, Phys. Rev. Lett. 88, 037901 (2002); Oliver Gywat, Guido Burkard, and Daniel Loss, Phys. Rev. B 65, 205329 (2002). 
[10] O. Benson, C. Santori, M. Pelton, and Y. Yamamoto, Phys. Rev. Lett. 84, 2513 (2000).

[11] R. G. DeVoe and R. G. Brewer, Phys. Rev. Lett. 76, 2049 (1996).

[12] T. Brandes and B. Kramer, Phys. Rev. Lett. 83, 3021 (1999).

[13] G. S. Solomon, M. Pelton, and Y. Yamamoto, Phys. Rev. Lett. 86, 3903 (2001).

[14] Paul S. Lee, Y. C. Lee, and C. T. Chang, Phys. Rev. A 8, 1722 (1973).

[15] J. M. Gérard, B. Sermage, B. Gayral, B.Legrand, E. Costard, and V. Thierry-Mieg, Phys. Rev. Lett. 81, 1110
(1998).

[16] S. Schneider and G. J. Milburn, Phys. Rev. A 65, 042107 (2002).

[17] Daniel Braun, Phys. Rev. Lett. 89, 277901 (2002).

[18] Z. Yuan, B. E. Kardynal, R. M. Stevenson, A. J. Shields, C. J. Lobo, K. Cooper, N. S. Beattie, D. A. Ritchie, M. Pepper1, Science 295, 102 (2002).

[19] L. Quiroga and N. Johnson, Phys. Rev. Lett. 83, 2270 (1999).

[20] S. Debald, T. Brandes, and B. Kramer, Phys. Rev. B 66, 041301(R) (2002). 